\title{
Aberration-Corrected STEM Analysis of Impurities in Cosmic Nanodiamonds and Synthetic Analogs
}

\author{
Rhonda M. Stroud ${ }^{1 *}$
}

1. Materials Science and Technology Division, US Naval Research Laboratory, 4555 Overlook Ave. SW, Washington, DC 20375, USA.

* Corresponding author: rhonda.stroud@nrl.navy.mil

Nanodiamond is an important material in planetary materials research because it was the first mineral phase identified as identified as the carrier of products of supernova nucleosynthesis pre-dating the formation of the Solar System [1], i.e., the first presolar grain phase. However, the size of the individual nanodiamonds, $\sim 2 \mathrm{~nm}$, is too small for isotopic measurements on an individual grain basis, and the abundance of the incorporated supernova impurities is such that the vast majority of the diamonds could have a solar system or local interstellar origin. Aberration-corrected scanning transmission electron microscopy of meteoritic nanodiamonds offers an alternative way to search for signatures of specific cosmic origins, through identification of elemental and structural variations at scales down to the single atom [2]. Parallel studies of doped synthetic nanodiamonds can provide insight into the diamond formation mechanisms and method of the impurity incorporation.

We examined nanodiamond samples with the Nion UltraSTEM-200X at the Naval Research Lab, equipped with a Bruker Xflash 100 energy dispersive X-ray (EDX) spectrometer, and Gatan Enfinium HR electron energy loss spectrometer (EELS). The nanodiamond samples included acid-resistant residues from the Allende meteorite, and doped nanodiamonds produced by high pressure / high temperature (HP/HT) phase transformation of carbon aerogels. The Nion was operated at $60 \mathrm{kV}$, with a nominal $0.15 \mathrm{~nm}, 50-100 \mathrm{pA}$ probe. Heating stage experiments to observe the transformation of the Allende nanodiamond residues with temperature were performed in the Nion with a Protochips Fusion system.

Meteoritic nanodiamond residues are often characterized by step-wise heating experiments, in which the gasses desorbed from the sample are elementally and isotopically characterized with mass spectrometry. Heating stage experiments in the UVH STEM can complement the mass spectrometry experiments, by identifying the temperature thresholds for physical changes to the diamond, as tracked with dark-field imaging, and C-K edge EELS (Fig. 1). For the Allende meteoritic nanodiamond sample, we observed a small pre-peak at $282 \mathrm{eV}$ (Fig. 1 red arrow), that we attribute primarily to NV centers. This peak dramatically decreased in intensity by $600^{\circ} \mathrm{C}$ due to the release of nitrogen, and possibly $\mathrm{He}$, from the diamonds. The increased contrast in the dark field image of the sample at $1000^{\circ} \mathrm{C}$ is due to the loss of impurities and surface carbon from the sample. By $1200^{\circ} \mathrm{C}$, a significant fraction, but not all, of the sample has graphitized, as shown in the EELS data by the shift in the $\pi^{*}$ peak position and intensity, and the decrease in the "dip" at $302 \mathrm{eV}$.

A combination of dark-field imaging, EELS and EDX observations of HT/HP synthetic nanodiamond demonstrate that Ar can be incorporated into the nanodiamond (Fig. 2) [3]. The Ar appears primarily to be distributed homogeneously, although some areas of high Ar concentration in trapped vesicles are also present. This suggests a possible mechanism for nanodiamond formation in space, through shock wave transformation of noble gas-rich organic ices, analogous to the laboratory transformation of argon-filled porous amorphous carbon aerogel. 


\section{References}

[1] R Lewis et al., Nature 326 (1987), p. 162.

[2] R Stroud et al., Applied Physics Letters 108 (2016), p. 163101.

[3] M. Crane et al., in review.

[4] The authors gratefully acknowledge funding from ONR/ NRL Core Program and NASA Emerging Worlds, assistance with heating holder from A. Shah, and samples from: (Allende) J. Greer and P. Heck at the Field Museum, (synthetic) M. Crane and P. Pauzauskie at the University of Washington.

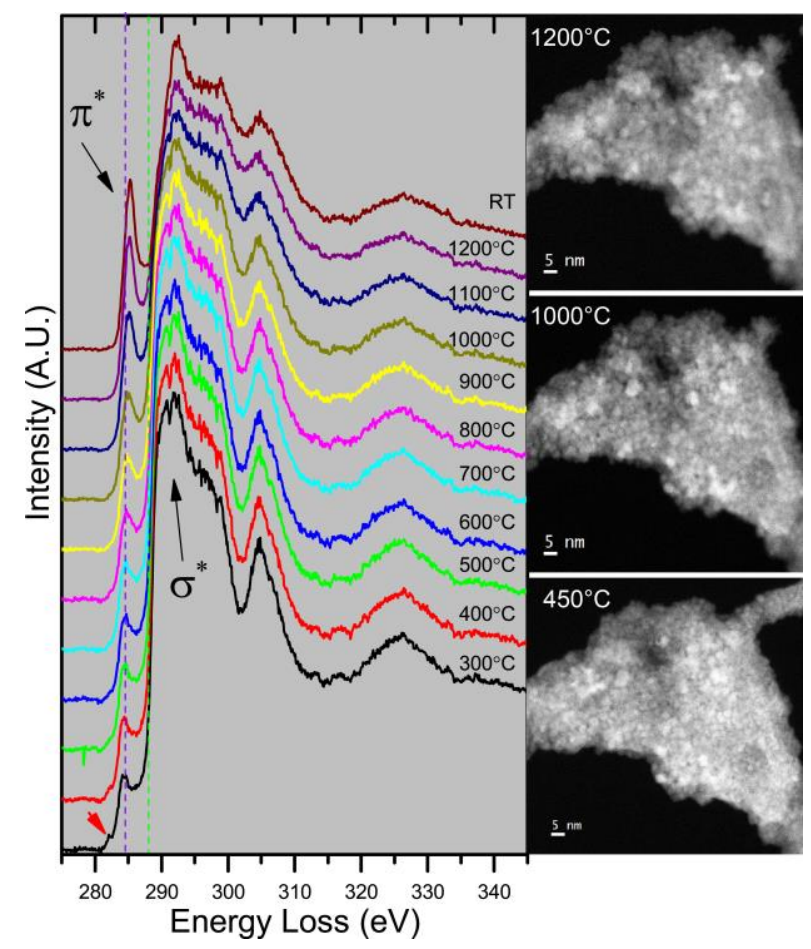

Figure 1. Carbon K-edge EELS and medium-angle annular dark-field STEM image of Allende nanodiamond heated in situ. The red arrow at the bottom left indicates a peak associated with $\mathrm{N}$ defects in the diamond lattice.
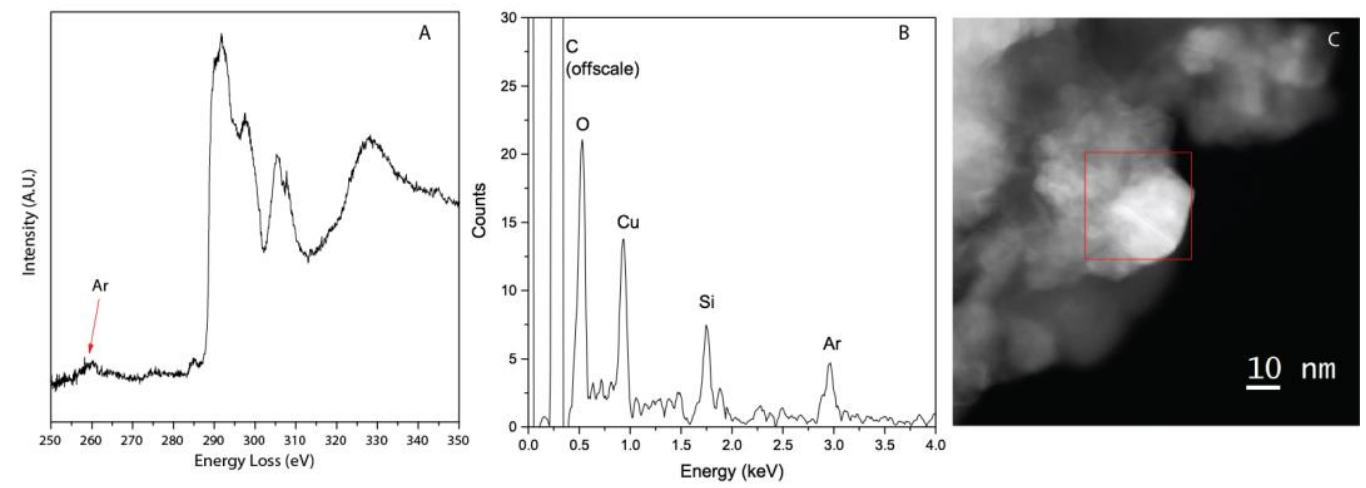

Figure 2. (A) C-K edge EELS and (B) EDXS of the area outlined in red in (C) medium- angle annular dark field image of Ar-doped synthetic HT/HP nanodiamond. 